\title{
EVALUACIÓN ACADÉMICA Y RELEVANCIA SOCIOPRODUCTIVA: LOS PROYECTOS DE DESARROLLO TECNOLÓGICOY SOCIAL (PDTS) COMO POLÍTICA CIENTÍFICA
}

\author{
ACADEMIC EVALUATION AND SOCIAL RELEVANCE: THE SOCIAL AND \\ TECHNOLOGICAL DEVELOPMENT PROJECTS (PDTS) AS A SCIENCE \\ POLICY INSTRUMENT
}

\author{
Judith Naidorf $\left.{ }^{*}\right)$ \\ Universidad de Buenos Aires, Buenos Aires, Argentina \\ Federico Vasen $^{(* *)}$ \\ Universidad Nacional de Quilmes; Universidad de Buenos Aires \\ Mauro Alonso ${ }^{(* * *)}$ \\ Universidad de Buenos Aires, Buenos Aires, Argentina
}

\begin{abstract}
Resumen: En este artículo presentamos resultados de investigación en torno al impacto de propuestas que objetivisan la investigación científica hacia formas de conocimiento que contribuyan a la solución de problemas sociales, demandas socio-productivas y prioridades definidas por los organismos de ciencia y tecnología. Indagaremos un nuevo instrumento de política científica que se propone en 2012. Se trata de los Proyectos de Desarrollo Tecnológico y Social (PDTS), enmarcaremos dicho análisis en algunos de los nuevos conceptos estelares (Braslavsky, 1999) como son las categorías de movilización del conocimiento y de innovación social, la innovación para la inclusión social y la innovación responsable.

Palabras claves: Proyectos de Desarrollo Tecnológico y Social (PDTS); movilización del conocimiento; innovación social.

Abstract: This paper presents the results of the research on the impact of research proposals aimed at solving social and industrial problems, according to priorities defined by the institutions of science and technology. We discuss a new science policy instrument that was first implemented in 2012: Social and Technological Development Projects (PDTS). We analyze this new initiative within the framework of the following categories: knowledge mobilization, social innovation, innovation for social inclusion and responsible innovation.
\end{abstract}

Keywords: Designing Technological and Social Development (PDTS); knowledge mobilization; social innovation.

(*) Doctora, Investigadora Adjunta de Instituto de Investigaciones en Ciencias de Educación, Universidad de Buenos Aires. E-mail: judithnaidorf@yahoo.com.ar.

(**) Doctor, Becario posdoctoral en el Instituto de Estudios sobre la Ciencia y la Tecnologia en la Universidad Nacional de Quilmes, Argentina. E-mail: fvasen@unq.edu.ar.

$(* * *)$ Mestre, Investigador del Instituto de Investigación en Ciencias de la Educación. E-mail: mauroralonso@gmail.com. Recibido en: 7.7.2015, aceptado en: 8.12.2015. 


\section{INTRODUCCIÓN}

La discusión sobre la relevancia socio productiva de la investigación científica formó parte de conjunto de temas centrales discutidos cuando la política científica se institucionalizó a mediados del siglo XX. El primer marco conceptual en el que se basaron estas políticas fue el llamado "modelo lineal". Este se basaba en un "contrato social" implícito entre ciencia y sociedad cuyo pilar se erguía en torno a la autonomía relativa de la comunidad científica (GUSTON, 2000). En este esquema, el Estado se comprometía a sostener económicamente el desarrollo y la promoción de ciencia básica y a brindarles autonomía a los investigadores para elegir los temas de investigación y para evaluar la calidad de las propuestas. Por su parte, estos se comprometían a ofrecer beneficios sociales "indeterminados". Bajo este modelo se construyeron instituciones como los "consejos de investigación" enfocados en la ciencia básica académica (RIP, 1996).

Como hemos señalado en otro trabajo, este contrato social fue evolucionando y la confianza en la capacidad de los investigadores de brindar esos productos sin interferir en su autonomía decayó (VASEN, 2011). La discusión por la relevancia y los resultados esperados se volvió más aguda y se comenzaron a definir tanto desde los gobiernos como desde los organismos de financiamiento de alcance regional y global así como desde fundaciones orientadas al financiamiento de actividades de investigación y de formación de posgrado prioridades y temas estratégicos para direccionar el financimiento de la ciencia de modo más directo a campos que podrían brindar conocimiento con potencial de aplicación en problemáticas sociales y productivas. En este artículo nos abocaremos específicamente al estudio de las primeras, es decir el análisis de las políticas científicas que orientan el financiamiento desde los gobiernos nacionales, en este caso una herramienta de política científica particularmente interesante y novedosa implementada en Argentina como son los Proyectos de Desarrollo Tecnológico y Social. Sin embargo, esta nueva tendencia ha encontrado límites y resistencias en los mecanismos clásicos de la comunidad científica para evaluar propuestas de financiamiento. Nos referimos a la evaluación por pares o peer review (HOLBROOK, 2010) de carácter disciplinar. Las prioridades y orientaciones políticas no han sido consideradas un criterio de peso en las evaluaciones centradas en la tradición bibliométrica.

En el presente trabajo nos proponemos analizar el desarrollo reciente de un nuevo instrumento de política científica en la Argentina impulsado desde el Ministerio de Ciencia Tecnología e Innovación Productiva (MINCTIP): los Proyectos de Desarrollo Tecnológico y Social (PDTS). Los PDTS son proyectos de investigación orientada que buscan promover el desarrollo de tecnologías asociadas a la resolución de una problemática social específico o al aprovechamiento de una oportunidad estratégica del mercado generando una vinculación más estrecha del potencial usuario de ese conocimiento en el proceso de investigación introduciendo métodos de evaluación que los distinguen de otros proyectos de investigación existentes. A diferencia de iniciativas previas, en este caso el foco de la intervención no está en la definición temática de las prioridades sino que ha surgido como una respuesta a las tensiones existentes entre las metodologías de evaluación de proyectos e investigadores de corte tradicional y la necesidad de contemplar las especificidades que requiere la valoración de aportes al desarrollo por parte de 
los científicos involucrados en este tipo de proyectos. Para contextualizar este cambio comenzaremos en la próxima sección por dar cuenta de las discusiones conceptuales en torno a los criterios de relevancia y a la investigación orientada por demandas extra académicas. A continuación, la sección tercera describe las discusiones sobre los distintos perfiles de investigadores que dieron lugar a la iniciativa de los PDTS. La sección cuarta describe las características de los PDTS y el marco normativo que los sustentan. Por último, la quinta sección da cuenta del avance de esta iniciativa, las características que asumen los proyectos incorporados y algunas de las discusiones que se generaron en torno a los mismos.

El artículo presenta una metodología mixta, a la vez cuantitativa y cualitativa a partir de un relevamiento de los PDTS en curso según la institución promotora y el área de conocimiento con objeto de describir la situación inicial de los primeros proyectos, el análisis de los documentos oficiales que dieron origen a la herramienta y conceptualizaciones producto de las primeras entrevistas realizadas a informantes clave y a directores de los primeros PDTS en curso del área de Ciencias Sociales y Humanas. El objetivo es mostrar las continuidades y rupturas acerca del modo de producción de conocimiento que la introducción de este nuevo instrumento de política científica habilita (haciendo especial foco en las modalidades de evaluación científica) tanto como los alcances, potencialidades y limitaciones de los PDTS como instrumento de política pública en ciencia y tecnología.

\section{CONCEPTOS PARA LA INVESTIGACIÓN ORIENTADA: ENTRE LAS NECESIDADES SOCIALES Y EL MERCADO}

El proceso de privatización de la ciencia que comenzó en la década de 1980 ha implicado un cambio en las "reglas de juego" (MIROWSKI, 2011). Mientras se suponía que los productos de la ciencia eran bienes públicos, a partir de legislación como la ley estadounidense Bayh-Dole de 1980, las universidades pueden comenzar a monetizar los resultados de su investigación. Esto produce a su vez un cambio en la cultura académica (NAIDORF, 2009), que se vuelve cada vez más proclive a ver con buenos ojos la comercialización de los resultados de su investigación. Slaughter y Leslie (1999), consideran a partir de ello el surgimiento de un "capitalismo académico" marcado por el incremento de vínculos de los académicos con el mercado. El capitalismo académico, señalan Slaughter y Leslie, se refiere al uso que las universidades hacen de su único valor activo real, el capital humano de sus académicos, con el propósito de incrementar sus ingresos. Afirmaba al respecto Eduardo Ibarra Colado (2003) que "tiene que ver con iniciativas y comportamientos económicamente motivados para asegurar la obtención de recursos externos".

En el plano internacional podemos afirmar el origen de la enunciación de las nuevas orientaciones de política científica se encuentra en el sector económico, a partir de la codificación mercantil del conocimiento (LLOMOVATTE; JUARROS; NAIDORF; GUELMAN, 2006). El paradigma de la economía de conocimiento intensivo revaloriza de manera particular la función de la investigación como generadora de dicho conocimiento. En este sentido, así lo enfatizaba el Informe del Banco Mundial sobre el Desar- 
rollo destinado al problema del conocimiento (BANCO MUNDIAL, 1998). Un año más tarde, la UNESCO convocó, en Budapest a la Conferencia Mundial de la Ciencia donde se emitió una declaración sobre la importancia del uso del saber científico, se buscó orientar el conocimiento al servicio del progreso, al desarrollo, a la paz y a su aplicación en función de permitir paliar el desequilibrio social y la exclusión (UNESCO, 1999).

A partir de estos postulados el desafío de la competitividad como estrategia para lograr el crecimiento económico fundado en el pensamiento neo-schumpeteriano alcanzó un carácter global (HARTMANN; PYKA; HANUSCH, 2010). La alianza entre desarrollo científico y desarrollo económico se constituyó en el actual patrón de producción de conocimiento a través políticas científicas que a nivel institucional, nacional e internacional han bregado por su dinamización e intensificación.

En este marco, en la visión del policy-maker, las iniciativas de política científica y tecnológica buscan algo más que acrecentar el stock de conocimientos disponibles sin un objetivo específico, - como podría sostener Polanyi (1968) -, se espera que la ciencia tenga una "función social" y una utilidad evidente. La pregunta "¿en qué consiste la función social de la ciencia?", si bien es claramente multívoca, ha sido respondida desde la política científica en las últimas décadas de un modo uniforme: en la innovación tecnológica. Muchos autores han descrito los cambios producidos en las prácticas científicas en respuesta a la necesidad de acercarlas a las prácticas innovadoras. Una de las propuestas más influyentes en este campo ha sido la de Gibbons et al. (1994) que distingue entre un modo de producción de conocimiento académico, disciplinar, en el que prima el control de calidad interno de los pares (el llamado "modo 1") y un modo emergente en el que se investiga en el "contexto de una aplicación", en forma transdisciplinar y con un control de calidad en el que participan actores externos (el "modo 2").

La propuesta de Gibbons ha sido criticada desde múltiples perspectivas, desde su falta de soporte en la teoría sociológica y en datos empíricos hasta la presencia de una intención normativa en sus aserciones (SHINN, 2002; GODIN, 1998). Más allá de las críticas, lo cierto es que el planteo de Gibbons acerca de la emergencia de un "nuevo modo de producción del conocimiento" fue muy influyente en los círculos de política científica (HESSELS; VAN LENTE, 2008). Paralelamente a Gibbons, otros autores, con orientaciones distintas, señalan la existencia de estas transformaciones; tal es el concepto de ciencia post-normal de Funtowicz y Ravetz (1993) que enfatiza la necesidad de participación de actores externos en la evaluación de actividades científicas con posible impacto ambiental y el enfoque de la triple hélice que plantea la existencia de una segunda revolución académica en las universidades en el marco de la cual éstas asumen como parte de su misión la contribución al desarrollo social y económico (ETZKOWITZ; LEYDESDORFF, 2000; ETZKOWITZ, 2008; SUTZ, 2000; 2005; VESSURI, 1996; VACCAREZZA, 2002; LLOMOVATTE, JUARROS, NAIDORF y GUELMAN, 2006).

En este escenario, a partir del año 2000, surge para la discusión de las políticas de investigación en ciencias sociales el concepto de movilidad o movilización del conocimiento (Knowledge Mobilization); esto es, el requerimiento de una producción de conocimiento "listo para la acción", que implica ir más allá de su difusión, en tanto también sería función del investigador encontrar caminos que enlacen la producción y 
la utilización del conocimiento producido. Sin embargo, dado que se han identificado dificultades en los canales de diálogo entre productores y usuarios de los conocimientos científicos, esta propuesta de movilidad o movilización del conocimiento es acompañada por lo que se denomina "interlocutores/gestores del conocimiento" o knowledge brokers que adquieren la forma de promotores/articuladores o matchmakers.

La categoría movilidad o movilización del conocimiento fue enunciada de forma reiterada por los entrevistados (investigadores en su rol de directores de proyectos, miembros de la gestión de la investigación en las universidades y evaluadores de proyectos de investigación en 2009) en Canadá. Esta denominación se enmarca en las tendencias que buscan orientar la investigación a prioridades establecidas principalmente por organismos estatales extrauniversitarios o por el parlamento, como es en el caso canadiense (NAIDORF, 2014).

La noción de movilidad o movilización del conocimiento que surge en principio para ser una categoría desde y para las ciencias sociales y las humanidades a diferencia de la mentada transferencia tecnológica, refiere a la puesta a punto del conocimiento para su aplicación práctica (LEVESQUE, 2009; SÁ et al, 2011; QI et LEVIN, 2013; FISHMAN, 2014). Mientras que la difusión (dissemination) no implica compromiso alguno con la responsabilidad en el proceso que conduce hacia el canal de llegada del conocimiento elaborado, la movilidad o movilización del conocimiento implica un paso intermedio entre el resultado arribado y su aplicación práctica. La metáfora utilizada para diferenciar difusión o diseminación y movilidad o movilización del conocimiento es la que se puede homologar al logro del crecimiento de una planta y la simple distribución de las semillas en una parcela de tierra. Mientras difusión o diseminación implica sólo arrojar las semillas a la tierra, la movilidad o movilización involucra otros procesos ligados al rastrillaje de la tierra, su fertilización, el regado, así como el compromiso con el objetivo de que al final lo plantado florezca.

\subsection{INNOVACIÓN SE DICE DE MUCHAS MANERAS}

En el debate previo existe un equívoco - o al menos una indeterminación - sobre el para quién se está disponibilizando o movilizando el conocimiento. Los modelos de triple hélice o modo - 2 manifiestan más explícitamente que de lo que se trata es de fomentar la vinculación entre la universidad y el mundo productivo, a través de la incentivación de la investigación contratada y la producción conjunta entre ambos actores de intangibles cuya propiedad es susceptible de ser protegida a través de patentes y otros mecanismos legales.

Lo que sucede es que estos marcos teóricos están asociados a una idea de "innovación tecnológica" de corte schumpeteriano, localizada en el sector privado, y que apunta al agregado de conocimientos como una forma de aumento de la competitividad económica. Sin embargo, en los últimos años, se ha comenzado a complejizar el concepto de innovación y aceptar que esa es solo una de las formas en las que este fenómeno tiene lugar. En algunos casos se ha sostenido que es necesario abandonar la noción de innovación, en la medida en que en su origen en los textos de Schumpeter ésta ya incorpora la noción de rentabilidad comercial y utilidad "para el mercado" (DIAS et DAGNINO, 
2007; HURTADO, 2014). Por otra parte, otros autores coinciden en la utilidad de la idea de innovación, pero sostienen que es necesario cambiar o ampliar su contenido (ARBER, GORDON, SLEIMAN, ALEGRÍA, DE MOORI KOENIG, 2014). En esta última línea, se encuentran fundamentalmente tres nuevos enfoques: la innovación para la inclusión social, la innovación social, y la innovación responsable.

Las iniciativas denominadas de "innovación para la inclusión social" provienen del campo latinoamericano y se orientan a generar, desde el campo universitario, conocimientos que sirvan a resolver necesidades específicas de los grupos desprotegidos y contribuyan de esta manera a la inclusión social, a través fundamentalmente de reducción de costos (AROCENA y SUTZ, 2012; ALZUGARAY et al, 2014). Esta perspectiva surge y desarrolla en el marco de un grupo localizado en la Universidad de la República en Uruguay bajo la dirección de Judith Sutz. Emparentado con esta perspectiva, grupos en Brasil y Argentina trabajan con el concepto de "tecnologías para la inclusión social". Thomas (2012) las define como una forma de diseñar, desarrollar, implementar y gestionar tecnología orientada a resolver problemas sociales y ambientales, generando dinámicas sociales y económicas de inclusión social y de desarrollo sustentable." Este enfoque enfatiza una dinámica bottom-up y de generación de dinámicas sociales y económicas autosustentables. Se relaciona en este sentido con la discusión global sobre grassroots innovations, que se centra en desarrollos surgidos por movimientos sociales y no motorizados en primer término por la academia o el sistema científico y tecnológico (SMITH; FRESSOLI; THOMAS, 2014). Recientemente, LALICS, el capítulo latinoamericano de la red Globelics sobre sistemas de innovación, ha difundido una declaración sobre los "aportes de la ciencia, la tecnología y la innovación a la inclusión social" (2014) en el cual se mencionan acuerdos básicos entre las distintas posturas de los académicos de la región sobre este tema, y dan la pauta de que próximamente se trabajará en profundizar los acuerdos y la construcción colectiva antes que las diferencias y matices entre los distintos enfoques en discusión.

En segundo lugar, también se encuentra la tendencia a ampliar el concepto de innovación a la noción de "innovación social" de modo de que no se focalice en los nuevos desarrollos tecnológicos, sino que incluya además las formas innovadoras de organización social, e incluya la figura de emprendedores sociales y no sólo tecnológicos. En este sentido, estos autores definen - basándose en Crozier y Friedberg (1993) - a la innovación social como "un proceso de creación en el curso del cual los miembros de una comunidad aprenden, es decir, inventan o establecen, nuevas formas de participar en el juego social de la colaboración y el conflicto, en una palabra se trata de una nueva práctica social". Cabe destacar que el concepto de innovación social puede no ser necesariamente normativo. Si bien algunos autores lo reservan para cambios sociales "positivos" o deseables, otros autores señalan que es necesario tener un concepto de carácter más analítico descriptivo, que pueda distinguir entre innovaciones o nuevas prácticas sociales "positivas" y aquellas que no son deseables (HOWALDT; SCHWARZ, 2010).

En tercer lugar, en el contexto europeo, ha ganado importancia el concepto de "innovación responsable", y se ha ubicado como uno de los temas clave en el componente de "ciencia con y para la sociedad" del programa de financiamiento de la Unión Europea 
Horizon 2020. Detrás de esta concepción está la idea de que se ha hecho hincapié en los efectos o impactos negativos de la I+D+i a través de las evaluaciones de riesgo e impacto ambiental, pero no se ha tematizado con igual nivel de complejidad cuáles son las consecuencias positivas de los nuevos conocimientos (VON SCHOMBERG, 2013). De acuerdo a esta perspectiva es necesario anticipar y dirigir la generación de conocimientos para que produzcan impactos positivos, y estos impactos no deben ser únicamente medidos en términos de mecanismos de mercado. Plantean que es necesario ir más allá de la idea de que una innovación ha tenido buenos efectos si ha tenido un éxito comercial. Por ello, la idea es poder tener desde el inicio en cuenta un conjunto de preguntas que permitan guiar responsablemente la innovación. Las dimensiones propuestas por Stilgoe, Owen y Macnaghten (2013) son anticipación, inclusión, reflexividad y capacidad de reacción. Se trata de poder acompañar el desarrollo de proyectos de innovación para que den cuenta de los intereses y valores de un conjunto amplio de actores.

Los Proyectos de Desarrollo Tecnológico y Social comparten parte de estas consideraciones al orientar sus objetivos (en tanto instrumento de política pública) y su desarrollo a la generación de conocimiento científico que se convierta o que sea insumo para la resolución de una problemática social. Al incluir conceptualizaciones novedosas como los de "institución adoptante" o "institución demandante" (consideraciones que desarrollaremos con mayor detenimiento en las secciones siguientes) se proponen articular activamente a los potenciales usuarios de los conocimientos a producir e incluirlos como parte fundamental del proceso de producción de conocimiento. De esta forma, los PDTS pueden considerarse como una propuesta innovadora que responde a la necesidad de consolidar una estrategia de orientación de políticas públicas en ciencia y tecnología hacia la consecución de metas sociales.

\subsection{POLÍTICAS ORIENTADAS EN ARGENTINA}

La política científica y tecnológica ha transitado por difíciles rutas y, como toda política pública, no se escinde de la orientación política general del Gobierno que la encamina ni de cómo se resuelven las correlaciones de fuerza entre Estado, sociedad y mercado (NAIDORF; PERROTTA; GOMEZ; RICCONO, 2015). Desde comienzos de los 2000 se observa en Latinoamérica una tibia aceptación a la definición de prioridades y temas estratégicos llevados adelante desde el estado (NAIDORF; PERROTTA, 2015), articulando las demandas por pertinencia y relevancia socio productiva de la investigación como ejes ahora nodales de las políticas públicas de ciencia y tecnología. Este área se ha conformado como un nuevo terreno de interés para el Estado desde mediados de los 2000 y los ejes de la política de ciencia y tecnología han sido: aumento del número de investigadores y becarios, recuperación de salarios y estipendios, incremento del financiamiento de proyectos en variadas modalidades, la repatriación de investigadores argentinos en el exterior y la elaboración del primer plan estratégico en CyT entre otras medidas (NAIDORF et al, 2015). La creación del Ministerio de Ciencia Tecnología e Innovación Productiva (Ley 26.388/07) como organismo articulador del complejo científico tecnológico argentino marca el renovado interés por la ciencia y la tecnología. En el año 2003 el gasto en actividades científicas y tecnológicas (ACyT) representaba el 0,46\% del producto interno bruto (PIB) mientras que para 2011, alcanzó el 0,73\% del 
PIB. Se observa un aumento en el gasto en ciencia y tecnología, que no solo ha acompañado el crecimiento del PIB, sino que también lo ha superado. Cabe destacar que el PIB de Argentina anual se ha incrementado a lo largo de los años: en el año 2003, según la CEPAL el PIB total anual - a precios constantes en dólares - fue de 153 897,9 millones de dólares, mientras que en el año 2011, ese mismo indicador alcanzó los 276 252,1 millones de dólares. A pesar del contexto fértil para el desarrollo de la actividad científica, sin embargo, se tiene aún como meta pendiente la consolidación de estrategias que sean capaces de romper con el modelo cientificista de producción científica que continúa siendo hegemónico dentro del campo científico local.

Las políticas científicas han impulsado instrumentos específicos para fomentar la investigación orientada a problemas de relevancia socioproductiva. Estas iniciativas pueden retrotraerse a la experiencia de los Programas Nacionales en la década de 1970 en Argentina y se continúan con la determinación de áreas prioritarias que fue incluyéndose en los diversos planes nacionales de Ciencia y Tecnología (GARGIULO; MELUL, 1992; EMILIOZZI, 2013). Si bien a nivel de las definiciones estratégicas, la presencia de prioridades para orientar la investigación no es nueva, lo que sí fue haciéndose cada vez más presente en los últimos años fue la necesidad de trasladar esos lineamientos estratégicos a instrumentos de política específicos. En este sentido se crearon, desde la Agencia Nacional de Promoción Científica y Tecnológica, líneas de subsidios orientadas como los Proyectos de Áreas Estratégicas (PAE) o los PICTO (Proyectos de Investigación Científico-Tecnológica Orientada). Más recientemente, a nivel de recursos humanos se crearon en el Consejo Nacional de Investigaciones Científicas y Técnicas (CONICET) convocatorias diferenciadas para becas de posgrado e ingreso a la carrera de de investigador ligadas a "temas estratégicos", definidos en el plan nacional de ciencia y tecnología así como convocatorias para becas posdoctorales PDTS.

Pero más allá de la existencia formal de mecanismos de acceso diferenciado al financiamiento, subsiste una dificultad central en el sistema nacional de ciencia y tecnología a la hora de que los proyectos orientados tuvieran continuidad y un mayor desarrollo. Esta dificultad radica en las características del sistema de evaluación de los logros y méritos de los investigadores que ha tendido a sobrevalorar los criterios bibliométricos y de algún modo a infravalorar o penalizar a los investigadores involucrados en proyectos centrados en la resolución de un problema, dado que en esta función concentran esfuerzos en otros aspectos y se priorizan otro tipo de acciones que en la evaluación no son ponderadas con la misma valoración que un paper. El sistema de evaluación es el núcleo del sistema científico (BIANCO et al, 2014) dado que un sistema de evaluación aceptado y consensuado por la comunidad científica es vital para el desarrollo de una ciencia rigurosa, útil y creativa para la comunidad nacional y regional.

En este punto nodal que constituye la evaluación se origina la iniciativa de política científica cuyo análisis desarrollaremos en lo que sigue de este trabajo: los Proyectos de Desarrollo Tecnológico y Social (PDTS), en la medida en que su foco no está en la asignación de un financiamiento específico sino en la generación y coordinación de instancias institucionales para un tipo de evaluación académica diferenciada que permita valorar las formas de conocimiento más aplicada, incluso cuando sus logros no se reflejen según las pautas tradicionales. 


\section{EL ORIGEN DE LOS PDTS: LAS DISCUSIONES EN TORNO AL SISTEMA DE EVALUACIÓN DE LA ACTIVIDAD DE INVESTIGACIÓN CIENTÍFICA}

A través de una serie de talleres, comisiones y emisión de documentos, se ha buscado en los últimos tiempos adaptar las reglas de evaluación de los investigadores hacia un sentido orientado a la innovación productiva. En nuestro caso la propuesta ha surgido principalmente de las políticas científicas gubernamentales para el propio sistema científico tecnológico y no como una demanda orgánica del empresariado argentino. La revisión histórica de las políticas científicas desde los años ochenta en Argentina permite no solo evaluarla como un proyecto inconcluso (HURTADO, 2010) sino como un sistema inserto en un mundo empresarial poco proclive a relacionarse con el sector científico (AZPIAZU, NOCHTEFF, 1994; NAIDORF, 2009).

Durante el año 2012 se ha llevado a cabo el: "Taller sobre Evaluación de la actividad de CyT en las Universidades Nacionales" en Vaquerías, Córdoba, 19 y 20 de abril. Este documento $^{(1)}$ y las discusiones suscitadas en 2012 marcan una puesta en consideración sobre las características actuales de la producción intelectual que nuestra investigación ha problematizado y tomado como objeto de indagación (NAIDORF y PEREZ, 2012).

Algunos de los considerandos enunciados en estos documentos tienen como fin sostener y argumentar las propuestas que se plasman allí. Es en dichos argumentos en los que vale la pena detenerse a analizarlos en tanto fundamentos de las propuestas.

En dicho taller se parte de destacar la valiosa experiencia del sistema científico argentino aunque se afirma que es hora de "incrementar la eficacia de sus aportes al desarrollo económico y social del país" (CIN, 2012, p. 2). La propuesta es complementar la capacidad bien desarrollada en investigación básica fundamentalmente proveniente de la oferta interna del propio sistema (o de los investigadores) con una investigación orientada que permita "atender las demandas que la sociedad presenta actualmente" (CIN, 2013, p. 2). Se afirma también que la falta de concordancia entre investigación básica y orientada así como su falta de complementariedad - en nuestro caso escasez de investigación orientada - "limita la significación social de ciencia y la tecnología." (CIN, 2012, p. 2).

Asimismo tanto en las evaluaciones del personal de CyT del CONICET como en los criterios adoptados por las universidad para evaluar a sus profesores e investigadores o desde el Programa de Incentivos de la Secretaría de Políticas Universitarias del Ministerio de Educación se "absolutizan categorías tales como excelencia, originalidad etc., y se subestima las actividades tecnológicas y la transferencia de conocimientos al sector público y privado, lo que se expresa en metodologías de evaluación de Recursos Humanos fundamentalmente basadas en criterios bibliométricos" (CIN, 2012, p. 3). Para ello la propuesta consensuada en dicho taller es la de "modificar los criterios de evaluación

(1) Documento del Consejo Interuniversitario Nacional: "Conclusiones del Taller sobre Evaluación de la actividad de CyT en las Universidades Nacionales Vaquerías, Córdoba, 19 y 20 de Abril de 2012. Disponible en: <http://goo.gl//eXacq>. 
predominantes en estas instituciones de forma tal de asignar los recursos necesarios en ambas áreas y, particularmente, de asegurar a los investigadores que quieran incursionar en proyectos orientados, objetivamente con menos posibilidades de publicar en revistas indexadas de alto índice de impacto, la continuidad en sus carreras de investigador y/o docente" (CIN, 2012, p. 3).

Estas decisiones, se afirma, son de carácter político y no solo técnico. La conclusión es que las actividades de Investigación Orientada (IO) son desalentadas y provocan desinterés por parte de los investigadores ya que no son reconocidas en las incorporaciones o promociones en sus carreras. El mayor grado de distorsión se da cuando se cumple la prima "publicar o perecer" (BARRENT, 1962; PARCHOMOVSKY, 2000; SKOLNIK, 2000) donde "la exigencia de publicar que demanda el sistema puede llegar a tener mayor prioridad que el objeto de los trabajos" (CIN, 2012, p. 4).

La propuesta no es sólo equiparar ambos abordajes u orientar más lo menos desarrollado (la IO) sino "poner en los primeros lugares de la escala aquellos elementos que más claramente den respuesta a las demandas del medio socioeconómico" (CIN, 2012, p. 4).

Al marcar las diferentes formas de evaluación se afirma que en el caso de la Investigación Básica (IB), los evaluadores son los especialistas del área mientras que en el caso de Investigación Orientada la evaluación requiere la visión in situ de los procesos de desarrollo, el seguimiento de la marcha del mismo, lo que implica no solo mayores recursos sino también la participación de otros actores, tales como los "no pares", actores del medio socio económico así como la opinión del propio demandante.

En este sentido se propone establecer algún sistema de equivalencias que permitan valorar la IO y que los investigadores involucrados no sean castigados por el sistema de promoción vigente.

En relación a la formación de doctores y a los posgrados que se desarrollan en las universidades, las propuestas han rondado en torno a "reformular nuestros posgrados de forma tal que tengan una mirada a las realidades locales y regionales, y un perfil profesional que los haga atractivos para ser incorporados por el medio y no necesariamente por las distintas instituciones del sistema de Ciencia y Tecnología" (CIN, 2012, p. 5).

Entonces y resumiendo las propuestas concretas refieren a:

- lograr un rápido incremento de la cantidad de proyectos de investigación orientados a la resolución de problemas de la sociedad

- generar mecanismos que faciliten la recepción de la demanda y su articulación con los grupos de investigación y desarrollo que podrían atenderla

- y promover la conformación interdisciplinaria y la participación de los sectores externos involucrados, fundamentalmente en el caso de que la transferencia de los resultados sea inmediata. (CIN, 2012, p. 6)

La experiencia de los encuentros en relación a construir y consolidar herramientas de funcionamiento para los proyectos de IO se encuadra en los lineamientos políticos 
que consideran a la ciencia como un vehículo para atender a problemáticas del medio socio-productivo. En este sentido, uno de los desafíos actuales que con la implementación de los PDTS se pretende atender es la conjugación de problemas sociales (traducidos en demandas o no) en objetos de investigación que logren convertirse a posteriori en respuestas concretas por parte de los académicos.

Los resultados de la implementación de este tipo de políticas científicas se verán en el largo plazo. Sin embargo se trata de una herramienta que ha convocado a más de 450 postulaciones de todas las áreas del conocimiento en su úlitma convocatoria, promovida por el CIN. Su estudio se presenta como altamente favorable ya que posibilita analizar no solo la modalidad de implementación de la herramienta y las primeras respuestas por parte de los científicos sino también contar con un registro de la evolución y adaptación de la misma en su funcionamiento.

Siguiendo a Albornoz (2014), las estrategias metodológicas de evaluación de política científica según el momento en que son aplicadas se distinguen en tres según sus momentos de aplicación: ex ante, intermedia y ex post.

Cada una de las estrategias posee beneficios y limitaciones propias y un análisis comprensivo de una herramienta de política científica no supone adoptar una metodología única de forma acrítica.

Los objetivos del plan de trabajo que desde el equipo coordinado por Naidorf (2011) estamos llevando adelante para el análisis integral de esta nueva herramienta de política científica se proponen emplear de forma conjunta los tres modelos de análisis centrándose en los objetivos que la herramienta se propone (ex ante), el desarrollo de la herramienta y su funcionamiento (intermedia) y los resultados obtenidos por la misma (ex post).

De esta forma el análisis combinará instrumentos de los tres momentos para relevar a la herramienta que suponen los PDTS en su funcionamiento analizando el desarrollo de los mismos en consonancia con sus objetivos iniciales y los procesos interactivos que los científicos y usuarios ponen en práctica durante el proceso de investigación.

\section{LA CRISTALIZACIÓN DE LA INICIATIVA: EL BANCO DE PROYECTOS DE DESARROLLO TECNOLÓGICO Y SOCIAL}

El documento de la comisión asesora sobre evaluación del personal científico y tecnológico del MINCYT denominado "hacia una redefinición de los criterios de evaluación del personal científico y tecnológico" y fechado el 15 de agosto de 2012, refrendado el 13 de septiembre y firmado el 4 de octubre de 2012, establece una serie de considerandos que es pertinente revisar en detalle.

En primer lugar es importante destacar que la Secretaría de Articulación Científico-Tecnológica es el ámbito que coordinó las actividades de esta comisión. En segundo lugar se hace referencia al $1^{\circ}$ taller convocado en 2011 donde se concluye que: no es pertinente utilizar los mismos sistemas de evaluación para el personal dedicado a la investigación básica (IB) que al dedicado a la investigación aplicada (IA) y al desarrollo 
tecnológico social (homologado este último a la IA) lo que redunda en una ponderación desequilibrada. También se establece la existencia de una distancia entre criterios de calidad académica - orientada hacia el modelo tradicional y lineal de la producción del conocimiento - y de relevancia y o pertinencia organizacional ${ }^{(2)}$.

Nuevamente en este documento se hace referencia a la definición política que implica este cambio que se propone y la denuncia de este desbalance en la evaluación de los investigadores involucrados en la IA así como la poca orientación del sistema vigente de estímulos e incentivos a ponderar dicha actividad.

Se afirma allí que la sobreponderación de los criterios de originalidad en detrimentos de los de aplicabilidad está afectando la capacidad del sistema científico tecnológico para atender a las demandas nacionales, regionales y locales de solución de problemas concretos. Otro aspecto que se destaca en el documento es el ligado a la preeminencia de la evaluación individual y la insuficiente valoración del trabajo en equipos.

Se acuerda a fin de dar respuesta a dichos problemas planteado, la elaboración de nuevos criterios comunes para la evaluación del personal dedicado al desarrollo tecnológico y social, con énfasis - argumentativo - en este último aspecto. La propuesta es entonces establecer un Banco Nacional de Proyectos que respondan a estas características y ofrecer de manera optativa a los investigadores involucrados en proyectos incluidos en el Banco una evaluación diferenciada.

Los Proyectos de Desarrollo Tecnológico y Social (PDTS) son proyectos de investigación que: 1) Están orientados al desarrollo de tecnologías asociadas a una oportunidad estratégica o a una necesidad de mercado o de la sociedad debidamente explicitada. Están dirigidos a la generación de productos, procesos, prospectivas o propuestas; 2) Se caracterizan por tener un plan de trabajo de duración acotada, con objetivos claros y factibles, actividades pasibles de seguimiento y evaluación, hitos de avance y resultados acordes con los objetivos. Deben incluir la indicación de grados de creación o de innovación de conocimientos; 3) Cuentan con una o más organizaciones públicas o privadas demandantes y con posibles adoptantes del resultado desarrollado. En este marco, puede ser demandante o adoptante una institución pública del sistema de ciencia y tecnología siempre que el proyecto se inserte en las líneas estratégicas de la institución; 4) Tienen una o más instituciones promotoras que proveerán su financiamiento; 5) Cuentan con una evaluación realizada por especialistas o idóneos que contemple: a) la factibilidad técnica y económico-financiera o equivalente; b) la adecuación de los recursos comprometidos (humanos, infraestructura y equipamiento, y financiamiento); y c) los informes de avances sobre la ejecución del proyecto en caso de que corresponda.

Vale destacar que no se prevé una línea de financiamiento adicional (hasta la convocatoria limitada a 3 proyectos por Facultad que abrió la UBA en 2013 y la convocatoria del CIN abierta en agosto de 2014) para el desarrollo de este tipo de proyectos porque tal como se establece en el punto 4 antes descripto estos proyectos deben incluir financiamiento del demandante. En ajustes a este nuevo instrumento se establecieron tres figuras: el adoptante (beneficiario de la investigación), el demandante (quien establece

(2) para el análisis en torno a la relevancia en la política científica ver Vasen, 2011; y sobre pertinencia ver Naidorf, 2011 
la necesidad de un proyecto en esta área) y el promotor (que aporta los recursos). El adoptante puede o no financiar el proyecto como contraparte.

La introducción de estas nuevas figuras analíticas se presenta a-priori como novedosa ya que supone la inclusión explícita de los potenciales usuarios del conocimiento a producir como parte del diseño y la construcción de la investigación.

\section{AVANCES EN LA IMPLEMENTACIÓN DE LOS PDTS}

\subsection{EL CAMBIO CULTURAL DESDE LAS UNIVERSIDADES: LA UBA COMO PROMOTOR DE LOS PDTS}

En una actividad denominada "La evaluación de la investigación en la Universidad Pública. Cambios y debates actuales en las Ciencias Sociales" convocada por el Programa de Estudios sobre la universidad pública del Instituto Gino Germani el 15 de noviembre de 2012 el Dr. Hugo Sirkin, entonces Secretario de Ciencia y Técnica de la Universidad de Buenos Aires manifestó que la crítica central que manifestaron en general los participantes miembros de cerca de cincuenta organizaciones fue:

- Que aunque el presupuesto para Ciencia y Técnica se ha elevado (a 0.67 porciento del PBI), no aumenta más por la incapacidad del sistema de demostrar la utilidad de la ciencia.

- Nos identificamos con un modelo de ciencia "que en lo esencial se mira al ombligo", "la ciencia no responde a las necesidades del momento", "se ha sobrevivido encerrándose como esta", la elección de temas de investigación "se basa en gustos y experiencias previas", no existen siquiera sólidos proyectos institucionales.

- Se acuerda la valoración sólida del sistema de investigación básica pero es necesario que una parte se reoriente (entre un $10 \%$ y un 15\%).

Estas afirmaciones son las que dieron lugar a la propuesta de los PDTS. Se destaca que no se trata solo de venta de servicios técnicos sino que son proyectos donde la creación de conocimiento es parte intrínseca de su sentido basal.

De la primera experiencia de apertura de una convocatoria intrainstitucional como fue el caso de la llevada a cabo en 2013 por Secretaría de Ciencia y Técnica de la UBA ${ }^{(3)}$ y en particular de su Dirección de Articulación Institucional e interdisciplinaria se extraen las siguientes caracterizaciones y conclusiones. Aunque la convocatoria fue publicitada por la SECYT las propuestas fueron elevadas por cada Facultad que a su vez realizó una pre-selección interna. Se presentaron ideas proyectos de las cuales solo una selección fue convocada a presentar el proyecto complete, cada propuesta fue acompañada por una carta compromiso del adoptante, las evaluación no llevaron puntaje y fueron cualitativas de valoración de factibilidad, que dado que no existe aún una comisión acreditadora de PDTS (a cargo de la Secretaría de Articulación del Ministerio de Ciencia Tecnología e Innovación Productiva los evaluadores fueron ad hoc propuestos para cada proyecto en particular. Por ello las evaluaciones estuvieron a cargo de una comisión cuatripartita

(3) ver: <www.uba.ar/archivos_secyt/image/2012-11-14_5778(1).pdf> 
compuesta por: un especialista en la disciplina, un miembro del Banco de evaluadores (este banco como dijimos aún no existe), la entidad adoptante y una figura denominada "idóneo" que podía haber sido alguien de fuera de la academia o lo que se denomina un "no par". Entonces la elaboración de una comisión evaluadora por proyecto fue una tarea compleja y nueva.

Lo notable es que los criterios de relevancia y pertinencia fueron definidos de manera explícita de la siguiente manera:

Criterio de relevancia: en el contexto del presente documento, se entiende que el criterio de relevancia es un concepto estrictamente político (en sentido amplio) que califica a un proyecto de I+D en función de los objetivos o fines a los que tiende y de los objetos a los cuales se aplica. La relevancia puede referir a la adecuación de los objetivos del proyecto a políticas públicas u objetivos estratégicos como así también vincularse a objetivos de política de sectores de la sociedad civil o a valores más o menos generalizados en la sociedad.

Criterio de pertinencia: en el contexto del presente documento, se entiende que la pertinencia considera la estrategia del PDTS en términos de su capacidad para resolver el problema identificado y la adecuación de los resultados esperados al uso concreto en el contexto local de aplicación.

De la implementación del Banco Nacional de PDTS y desde la Secretaría de Articulación se extraen algunas conclusiones que son las siguientes:

- Que el Banco PDTS fue creado como una herramienta de cambio respecto de las prácticas de evaluación y que en ese sentido no ha tomado (ni pretende tomar por lo que se afirma) un carácter masivo ni de progresiva ampliación

- Que en el primer año de la implementación (2013) de la opción para los investigadores de CONICET de ser evaluados en su informe anual (para investigadores asistentes) o bianual (para el resto de las categorías) de producción de conocimiento según se estima que un 20 porciento de los investigadores involucrados en proyectos pertenecientes al Banco optaron por una evaluación de su desempeño ad hoc en lugar de según el criterio clásico disciplinar

En este sentido y en virtud de tratarse de Secretarías que se define como "hacia adentro" o de articulación intra e interinstitucional y no se asumen estas medidas como herramientas estratégicas de la planificación de la política científica aunque creemos que en la práctica lo es.

La convocatoria abierta en 2014 denominada "PDTS-CIN" tal como lo preveíamos ha provocado el interés de más investigadores de todas la universidades y por su presentación se ajusta a la mirada antes dicha y más amplia que la herramienta en sí que es la configuración de un nuevo perfil de investigador orientado a la solución de problemas sociales concretos. Estos proyectos como se dijo no son de extensión, son de investigación y no es estrictamente transferencia tecnológica o un servicio que se presta sino que son proyectos de investigación con adoptante identificado.

El Documento II de la Comisión Asesora, previamente mencionado en nota al pie define a la entidad adoptante como: "al beneficiario o usuario en capacidad de aplicar 
los resultados desarrollados en el marco de los PDTS". De esta forma, al deber identificar al potencial usuario del conocimiento a generar, los PDTS adquieren una estrategia que los distingue de otros tipos de proyectos de investigación orientada.

\subsection{LAS CARACTERÍSTICAS DE LOS PROYECTOS ACREDITADOS EN EL BANCO NACIONAL DE PDTS}

Para conocer más en detalle cómo ha evolucionado la iniciativa hasta el momento, nos permitimos realizar un breve análisis de las características de los proyectos acreditados en el Banco Nacional de PDTS (BNPDTS). En primer lugar se destaca que de los 105 proyectos presentes en el BNPDTS al 27/08/14, 60 (57\%) son ejecutados por una única institución del sistema científico (universidades u organismos), mientras el 43\% restante están conformados por una red de instituciones (Gráfico 1).

En cuanto al tipo de institución participante, puede apreciarse que las universidades participan del $71 \%$ de los PDTS, el CONICET del 47\%, y otros organismos de CyT del 24\% (Gráfico 1). Hay un 42\% de proyectos que solo son ejecutados por universidades, sin la participación de otros organismos de CyT. Si bien pueden encontrarse diferencias entre los grados de participación de las distintas instituciones, también puede afirmarse que las instituciones más importantes del sistema científico están participando en la iniciativa, lo cual da cuenta de un buen grado de aceptación y apropiación de este nuevo tipo de proyectos.

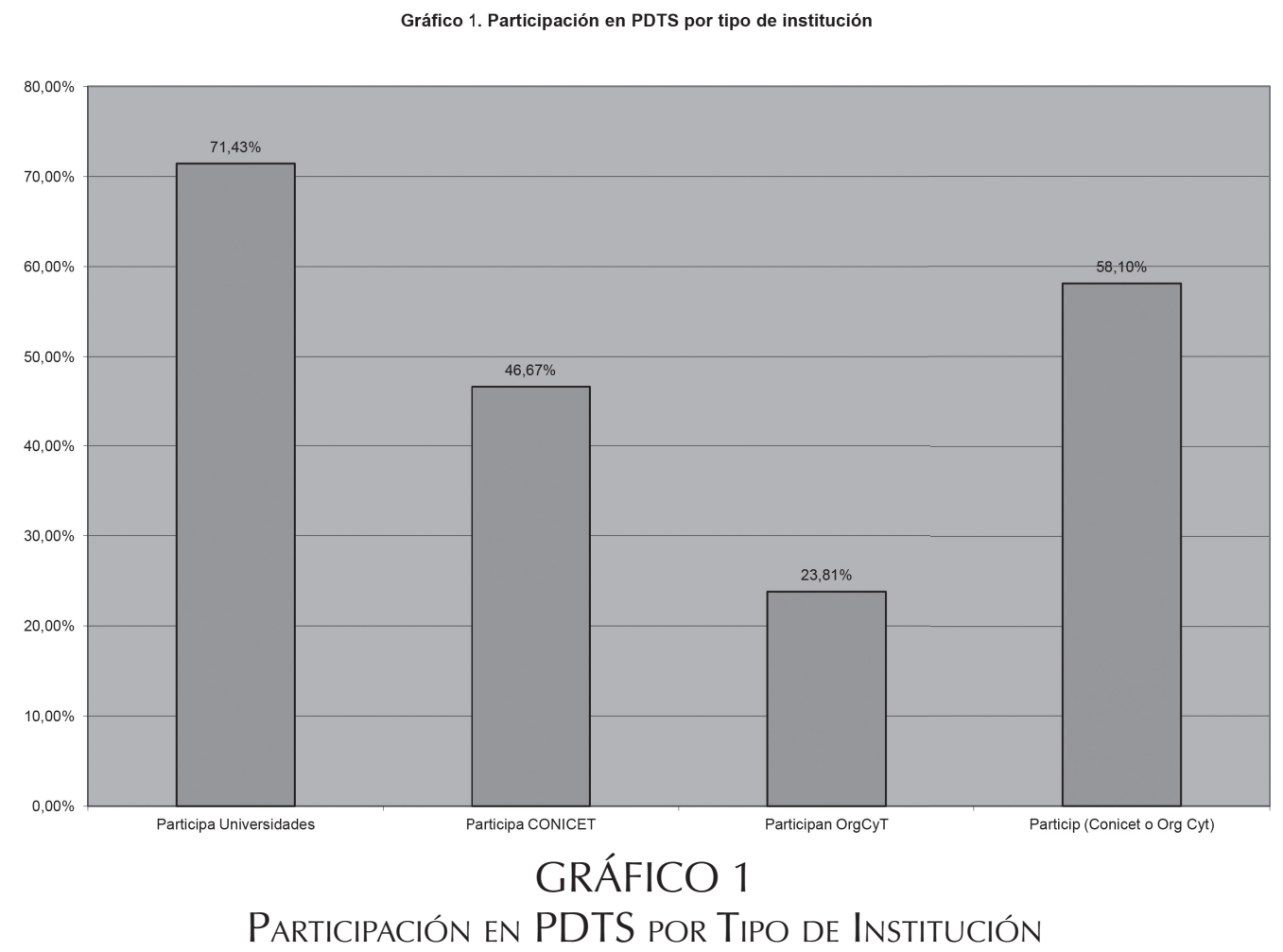

Fuente: Elaboración Propia en base a Datos del Banco Nacional de Proyectos de Desarrollo Tecnológico y Social. 


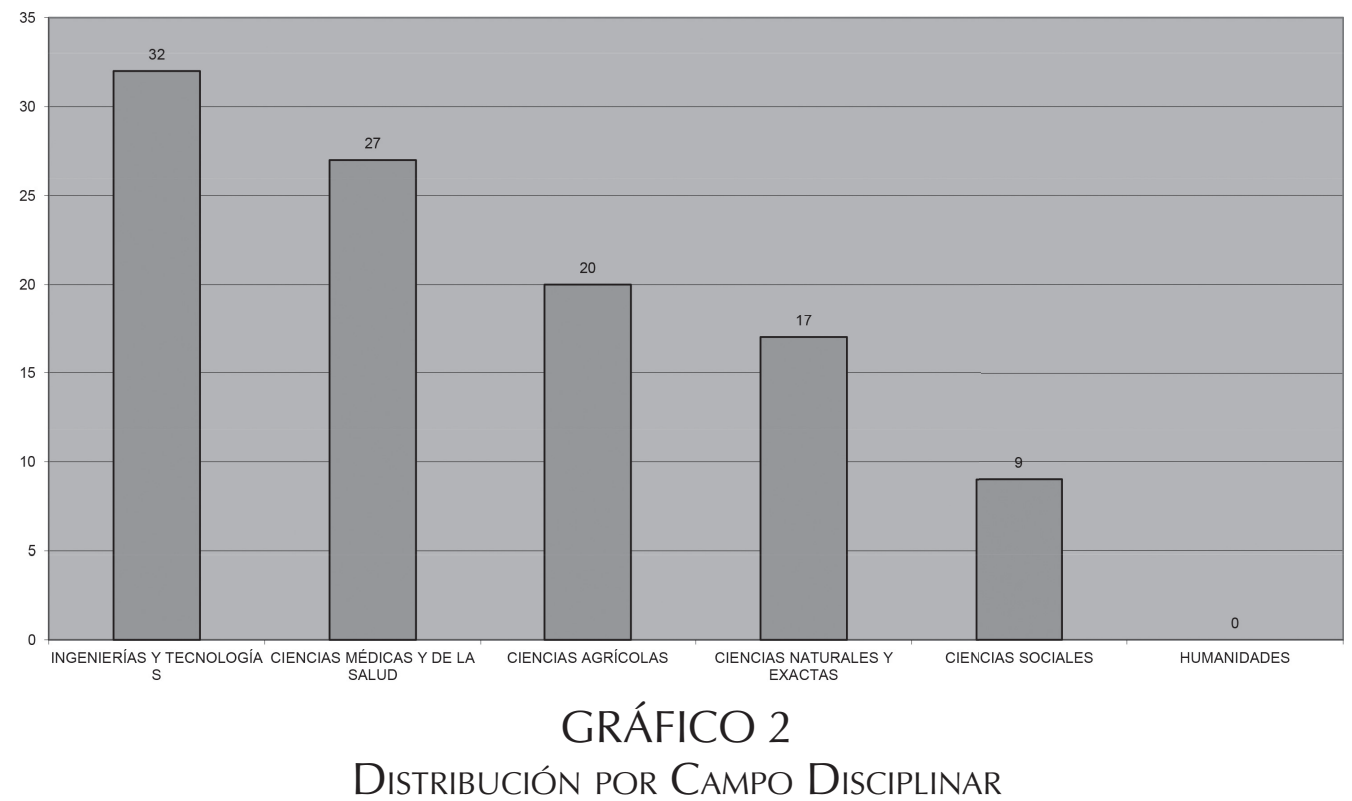

Fuente: Elaboración Propia en base a Datos del Banco Nacional de Proyectos de Desarrollo Tecnológico y Social.

En lo que hace a la distribución por disciplinas, cabe destacar que las ingenierías son las privilegiadas con el 31\%, seguidas por las ciencias médicas (26\%) y las agrícolas (19\%). Las ciencias exactas (16\%), sociales (9\%) y las humanidades (sin proyectos) son las menos representadas (Gráfico 2). Esto puede indicar un sesgo - previsible - hacia las disciplinas más aplicadas. Mientras las ciencias exactas y naturales lideran habitualmente los ránkings en los otros instrumentos de promoción de la investigación científica (representaron por ejemplo el 45\% en PICT2011-2012), en este caso el mayor número de proyectos no se da en las áreas ligadas a la investigación orientada por la curiosidad (sea en ciencias "duras" o "blandas"), sino en aquellas áreas orientadas por necesidades concretas. Se destaca la ausencia de proyectos PDTS en el caso de las humanidades, lo cual puede deberse a la dificultad de estas disciplinas para pensar su impacto social en los términos en que esta categoría es planteada por la política científica (hemos discutido este problema en Vasen, 2012). Por otra parte, cabe destacar que el BNPDTS solo ofrece una información sobre una única disciplina por proyecto, lo cual no permite apreciar si existe una conformación interdisciplinaria de los grupos de trabajo. Suponemos que existe en estos proyectos un grado mayor de interdisciplina que en los proyectos tradicionales de investigación, ya que esto se encuentra explícitamente sugerido por los documentos base de PDTS, pero lamentablemente - al igual que respecto de los adoptantes -, la información referida a esos puntos no está disponible en el BNPDTS.

\section{CONCLUSIONES}

En el documento del MINCyT se define a los PDTS como aquellos orientados a la elaboración de tecnologías tendientes a vinculación entre los demandantes o adoptantes 
de las mismas. La tendencia a vincular a las investigaciones al medio socio-productivo se emparentan con las propuestas de vinculación universidad-empresa de inicio de los ochenta que en su momento no prosperó y otras propuestas pasadas ligadas a la investigación orientada. Dado que estas cuestiones tendrán un rol central y un impacto relevante en la cultura académica y que se enlaza con los pretéritos temas de investigación, una de las funciones de análisis para los próximos años se debería orientar a contribuir a la mejor comprensión de estos procesos de recambio, marcar opciones de prospectiva a través del enriquecimiento de mirada comparada respecto de los procesos ocurridos en otros países.

En resultados de investigación y en nuestras tesis doctorales habíamos identificado incentivos para la participación en proyectos de transferencia tecnológica y de innovación a través de dos tipos de instrumentos: La resolución del CS de la UBA 1195/87 del 15 de abril de 1987 y la Ley de Innovación Tecnológica de 1991. Ambas normativas permiten la percepción de un emolumento adicional a aquellos investigadores involucrados en actividades que produzcan beneficios económicos a la universidad. Ambos instrumentos buscaron cambiar la cultura académica de la universidad y orientarla hacia una mayor interacción con el sector productivo.

Webster y Etzkowitz (1991) argumentan que estas nuevas formas de relaciones con la empresa producen la comercialización de las actividades universitarias. Ésta se genera desde dos ángulos diferentes: la que es promovida por las universidades mismas, que da origen a una empresarialidad académica a la que se denomina "comercialización en la academia", y la que promueve la empresa, denominada "comercialización de la academia", y que tiene como característica fundamental la participación directa y formal de los empresarios en organismos o mecanismos creados en el interior de las universidades para orientar sus actividades hacia las necesidades del sector productivo.

Las principales características de lo que hemos dado en llamar las Actuales Condiciones de Producción Intelectual (ACPI) están marcadas por: 1) aumento de la competencia entre pares; 2) la hiperproductividad medida en términos cuantitativos; 3) la tensión entre la individualización de la evaluación y la promoción del trabajo grupal y en redes; 4) la burocratización de las actividades de investigación; 5) la labor a corto plazo y por proyectos específicos; 6) la tensión entre la hiperespecialización y los abordajes inter, multi y transdisciplinario; 7) la búsqueda permanente de subsidios a la investigación y 8) las tensiones existentes en torno a realizar investigaciones pertinentes (NAIDORF; PEREZ MORA, 2012).

Estos documentos aquí analizados ponen en cuestión estas características de las condiciones de producción intelectual y se posicionan en el cuestionamiento de algunas de las mismas.

Estos postulados en torno a formas alternas de evaluaciones es un punto a favor en la revisión de los criterios tradicionales de la valoración del trabajo académico. Como ejemplo de ello en la reciente evaluación de las carreras de investigador científico se ha incluido un apartado que enunciaba que se agregó una sección para registrar la participación en PDTS y permite la opción de que el informe reglamentario de los investigadores sea evaluado por la Comisión Asesora de su disciplina o por la Comisión Asesora de Tecnología para PDTS especialmente creada para tal fin ${ }^{(4)}$.

(4) (http://pdts.mincyt.gov.ar/proyectos/) 
Sin embargo la homologación entre atención a las demandas del sistema productivo y la respuesta a los problemas sociales sigue generando imprecisiones y equívocos que no logran establecer una opción política a favor de cumplir el retrasado anhelo de diseñar un sistema orientado a resolver las necesidades regionales, nacionales y locales, cuestionar las desigualdades del sistema y poner la ciencia al servicio de la sociedad que la sostiene.

La introducción de la categoría de movilidad o movilización del conocimiento para el caso específico de las ciencias sociales, implica comprender en términos comparados esta contradicción no resuelta entre la aplicabilidad y utilidad del conocimiento y las tareas ineludibles de comprensión y transformación de los procesos sociales que le incumben a la ciencia intrínsecamente. La complementariedad de estas visiones y su complejización evitarían entonces opciones maniqueas y descalificatorias para uno u otro abordaje en función de una orientación políticas de las políticas científicas tanto en los niveles institucionales, nacionales y regionales.

Un seguimiento con perspectiva de historicidad de los PDTS podría dar una pista de que aspectos están orientados a una genuina solución de problemas sociales y cuales son proyectos en los que sólo las empresas se benefician aumentando su rentabilidad pero sin un impacto real en la calidad de vida del colectivo. Para profundizar estos últimos impactos, sería importante incluir dentro de la evaluación de los PDTS criterios asociados a marcos como las "tecnologías para la inclusión social", la "innovación social" o la "innovación responsable" que no sólo tematizan la aplicabilidad del conocimiento sino también su orientación a metas que se orientan primordialmente al desarrollo social antes que al crecimiento económico y la generación de riqueza.

A la hora de evaluar las características novedosas o particularmente interesantes de la iniciativa de los PDTS puede señalarse que las mismas se encuentran a primera vista antes en el nivel de la implementación que en el nivel de las ideas que la fundamentan. Es decir, fundamentalmente los PDTS implican un cambio en las formas de evaluación que tienden a acortar el hiato existente entre la definición de los objetivos estratégicos de las políticas y las formas concretas en que esas políticas son implementadas. Al involucrarse con los procesos de evaluación académica, ponen su foco en un punto que es habitualmente "ciego" para las políticas, una suerte de "caja negra" que tradicionalmente imprimía a los resultados de las evaluaciones un sesgo "academicista" contrario a la orientación de la investigación a temas estratégicos. A su vez, los esfuerzos de coordinación interinstitucional que se ven en la iniciativa son en sí mismos un avance fundamental dentro del sistema científico-tecnológico argentino, que carece de instancias consolidadas para la armonización de criterios. En este sentido, una situación particularmente conflictiva que esta iniciativa puede contribuir a destrabar es la de los investigadores de CONICET con lugar de trabajo en universidades, que debían responder a expectativas institucionales diferentes. Incluso cuando las universidades tienen criterios de evaluación más plurales, la pertenencia de los investigadores a la carrera de CONICET los obliga a adecuarse a las pautas bibliométricas tradicionales de este último organismo. Entonces, la pertenencia de un investigador a la dinámica de los PDTS permitiría en un futuro que los investigadores no sean castigados por ninguna de las instituciones en las que trabajan por involucrarse en proyectos orientados al desarrollo tecnológico y social. 


\section{BIBLIOGRAFÍA}

ALBORNOZ, Mario. Evaluación en ciencia y tecnología. En: Revista Perspectivas Metodológicas, v. 1, n. 3, 2014.

ALZUGARAY, Santiago; BIANCO, Mariela; GOÑI, María; MEDEROS, Leticia; SUTZ, Judith y ROBAINA, Sofía. Investigación e innovación orientadas a la inclusión social: análisis reflexivo de un proceso experimental. En: BIANCO, Mariela y SUTZ, Judith, coords. Veinte años de políticas de investigación en la Universidad de la República. Aciertos, dudas y aprendizajes. Montevideo: CSIC/ Trilce, p. 187-208, 2014.

ARBER, Gustavo; GORDON, Ariel; SLEIMAN, Cecilia; ALEGRIA, Daniela; DE MOORI KOENIG, Virginia. Innovación social, ciencia y tecnología para el desarrollo inclusivo. Disponible en: <http:// www.politicassociales.gov.ar/odm/ODM_SerieDocumentoTrabajo/ODM_SerieDocumentoTrabajoN5.pdf>. Fecha de consulta: 17 de sept. de 2014.

AROCENA, Rodrigo; SUTZ, Judith. Research and Innovation Policies for Social Inclusion: an Opportunity for Developing Countries. En: Innovation and Development, v. 2, n. 1, p. 147-158, 2012.

AZPIAZU, Daniel y NOCHTEFF, Hugo. El desarrollo ausente. Restricciones al desarrollo, neoconservadorismo y elite económica en la Argentina. En: Ensayos de Economía Política. Buenos Aires: Editorial Tesis/Norma, 1994.

BANCO MUNDIAL. El conocimiento al servicio del desarrollo, Washington: Banco Mundial, 1998.

BIANCO, Mariela; GRAS, Natalia; SUTZ, Judith. Reflexiones sobre la práctica de la evaluación académica. En: BIANCO, Mariela; Sutz, Judith (Coords.) Veinte años de políticas de investigación en la Universidad de la República. Aciertos, dudas y aprendizajes. Montevideo: CSIC/Trilce, p. 209-233, 2014.

BARRETT, Norman. Publish or perish. Thorac Cardiovasc Surg. v. 44, p. 167-79. London, UK; Thorax, 1962.

BRASLAVSKY, Cecilia. Los conceptos estelares de la agenda educativa en el cambio de siglo. En: Re-haciendo escuelas. Un nuevo paradigma en la educación latinoamericana. Buenos Aires: Santillana, 1999. COMITÉ INDEPENDIENTE DE CIENTÍFICOS, UNIVERSITARIOS Y PROFESORES (1973). Por una política científica y tecnológica nacional. En: Revista Ciencia Nueva, n. 22, p. 39. Buenos Aires: Ciencia Nueva,1973.

CROZIER, Michel. FRIEDBERG, Echard. Die Zwänge kollektiven Handelns. Über Macht und Organisation. Frankfurt: Hain, 1993.

DIAS, Rafael; DAGNINO, Renato. A política científica e tecnológica brasileira: três enfoques teóricos, três projetos políticos. En: Revista de Economia, v. 33, n. 2, p. 91-113. Curitiba: Universidade Federal do Paraná, 2007.

ETZKOWITZ, Henry. The Triple Helix. University-Government-Industry Innovation in Action. Nueva York: Routledge, 2008.

ETZKOWITZ, Henry; LEYDESDORFF, Loet. The dynamics of innovation: from National Systems and "Mode 2" to a Triple Helix of university-industry-government relations. En: Research Policy, v. 29, p. 109-123. Pensilvanya: Elsevier, 2000.

FISHMAN, Gustavo (The Equity Alliance). Mobilizing educational research for equity, 2014. Disponible en: <http://vimeo.com/equityalliancehttp://vimeo.com/87115297>.

FUNTOWICZ, Silvio; RAVETZ, Jerome. Science for the Postnormal Age. Futures, v. 25, p. 735-755. London: Butterworth - Heinemann Ltd, 1993.

GARGIULO, Gerardo; MELUL, Sara. Análisis de los Programas Nacionales de Investigación de la Secretaría de Ciencia y Técnica. En: OETIZA, Enrique (Dir.) La política de investigación científica y tecnológica en Argentina. Historia y Perspectivas, p. 317-338. Buenos Aires: CEAL, 1992. 
GIBBONS, Michael; LIMOGES, Camille; NOWOTNY, Helga; SCHWARTZMAN, Simon; SCOTT, Peter; TROW, Martin. The New Production of Knowledge: The Dynamics of Science and Research in Contemporary Societies. London: SAGE, 1994.

GIBBONS, Michael. Pertinencia de la educación superior en el siglo XXI. Ponencia presentada en: Conferencia Mundial sobre Educación Superior. París: UNESCO, 1998.

GODIN, Benoit. Writing performative history: the new new Atlantis? Social Studies of Science, v. 28, n. 3, p. 465-483. London: Sage Publications, 1998.

GUSTON, David. Between Science and Politics. Cambridge: Cambridge University Press, 2000.

HARTMANN, Dominik; PYKA, Andreas; HANUSCH, Horst. Applying Comprehensive Neo-Schumpeterian Economics to Latin American Economies. En: Structural Change and Economic Dynamics, v. 21, n. 1, p. 70-83. Georgia: Georgia Institute of Technology, 2010.

HESSELS, Laurens y VAN LENTE, Harro. Re-thinking new knowledge production: a literature review and a research agenda. En: Research Policy, v. 37, p. 740-760. Pensilvanya: Elsevier, 2008.

HOLBROOK, J.Britt. Peer Review. En: MITCHAM, Carl;,THOMPSON KLEIN, Julie; FRODEMAN, Robert (Eds.) The Oxford Handbook of Interdisciplinarity, p. 321-332. Oxford: OUP, 2010.

HOWALDT,Jürgen; SCHWARZ, Michael. Social innovation: concepts, research fields and international trends. Dortmund: IMO, 2010.

HURTADO, Diego. La Ciencia Argentina: un proyecto inconcluso 1930-2000. Buenos Aires: Edhasa, 2010. HURTADO, Diego. El fetiche de la innovación. Tecnología Sur-Sur, 11 de sept. de 2014. Disponible en: <http://www.unsam.edu.ar/tss/el-fetiche-de-la-innovacion/>. Consultado: 17 de sept. de 2014.

IBARRA COLADO, Eduardo. Capitalismo académico y globalización: la universidad reinventada. En: Educação \& Sociedade. v. 24, n. 84, p. 1059-1067. Campinas: Center of Studies Education and Society - CEDES, 2003.

LALICS - Latin American Network on Learning, Innovation and Competence Building. Declaración "Aportes de la ciencia, la tecnología y la innovación a la inclusión social", 2014. Disponible en: <http:// lalics.org/images/fondo_banner/Declaraci\%C3\%B3n_LALICS.pdf $\geq$.

LEVESQUE, Peter. Knowledge Mobilization Works. Ottawa, 2009. Disponible en: <www.knowledgemobilization.net $\geq$, fecha de consulta: 13 de jun. de 2009 .

LLOMOVATE, Silvia; JUARROS, Fernanda; NAIDORF, Judith; GUELMAN, Anahí. La vinculación Universidad - empresa: miradas críticas desde la universidad pública. Buenos Aires: Miño y Dávila Editores, 2006.

MIROWSKI, Philip. Science-mart. Privatizing American Science. Cambridge: Harvard University Press, 2011.

NAIDORF, Judith. Los cambios en la cultura académica. Buenos Aires: EUDEBA, 2009.

NAIDORF,Judith. Criterios de relevancia y pertinencia de la investigación universitaria y su traducción en forma de prioridades. En: Revista electrónica de la Asociación de Sociología de la Educación, v. 4, n. 4, p. 48-58. Madrid: Asociacion de Sociologia de la Educación, 2011.

NAIDORF, Judith; PEREZ MORA, Ricardo (Coords.) Las condiciones de producción intelectual de los académicos en Argentina, Brasil y México. Buenos Aires: Miño y Dávila, 2012.

NAIDORF, Judith. Knowledge utility: From social relevance to knowledge mobilization. En: Education Policy Analysis Archives, v. 22 n. 89. Arizona: Arizona State University, 2014.

OTEIZA, Enrique (Dir.) La política de investigación científica y tecnológica en Argentina. Historia y Perspectivas. Buenos Aires: CEAL, 1992.

PARCHOMOVSKY, Gideon. Publish or Perish. Michigan Law Review v. 98, n. 4, p. 926-952. Michigan: First Impressions, 2000. 
POLANYI, Michael. The Republic of Science. Its Political and Economic Theory. En: Shils, Edward (Ed.) Criteria for Scientific Development. Public Policy and National Goals, p. 1-21. Cambridge: MIT Press, 1968.

RIP, A. La república de la ciencia en los '90. En: Revista Zona Abierta, n. 75/76. Madrid: Fundación Pablo Iglesia, 1996.

QI, Jie; LEVIN, Ben. Assessing Organizational Efforts to Mobilize Research Knowledge in Education. En: Education Policy Analysis Archives, v. 21, n. 2. Arizona: Arizona State University, 2013.

SÁ, Creso; LI, Shannon; FAUBERT, Brenton. Faculties of education and institutional strategies for knowledge mobilization: An exploratory study. En: Higher Education: The International Journal of Higher Education and Educational Planning, v. 61 n. 5, p. 501-512. New York: Springer, 2011.

SHINN, Terry. La Triple Hélice y la Nueva Producción del Conocimiento enfocadas como campos socio-cognitivos. En: Revista Redes, v. 9, n. 18. Buenos Aires: Instituto de Estudios Sobre Ciencia y Tecnologia, UNQ, 2002.

SKOLNIK, Merril. Does counting publication provide any useful information about academic performan$c e$ ? En: Teacher Education Quaterly. San Francisco, California: Council on Teacher Education, 2000. SLAUGHTER, Sheila; LESLIE, Larry. Academic Capitalism. Baltimore: Johns Hopkins University Press, 1999.

SMITH, Adrian; FRESSOLI, Mariano; THOMAS, Hernán. Grassroots innovation movements: challenges and contributions. En: Journal of Clean Production, v. 63, p. 114-124. Pensilvanya: Elsevier, 2012.

STILGOE, Jack; OWEN, Richard; MACNAGHTEN, Phil. Developing a framework for responsible innovation, En: Research Policy, v. 42, p. 1568-1580. Pensilvanya: Elsevier, 2013.

SUTZ, Judith. The role of universities in knowledge production. SciDev, abr. 2005. Disponible en: $<$ http://www.scidev.net/en/policy-briefs/the-role-of-universities-in-knowledge-production-.html>. SUTZ, Judith. The university industry government relations in Latin America. En: Research Policy, v. 29, p. 279-290. Pensilvanya: Elsevier, 2000.

THOMAS, Hernán. Tecnologías para la inclusión social en América Latina: de las tecnologías apropiadas a los sistemastecnológicos sociales. Problemas conceptuales y soluciones estratégicas. En: THOMAS, Hernán FRESSOLI, Mariano;SANTOS, Guillermo (Orgs.) Tecnología, Desarrolloy Democracia. Nueve estudios sobre dinámicas socio-técnicas de exclusión/inclusión social. Buenos Aires: MINCyT-UNQ, 2012.

UNESCO. Draft, Declaration on Science and the Use of Scientific Knowledge, World Conference on Science, Budapest, 1999.

UNZUE, Martín; EMILIOZZI, Sergios. Universidad y políticas públicas ¿En busca del tiempo perdido? Argentina y Brasil en perspectiva comparada. Buenos Aires: Imago Mundi, 2013.

VACCAREZZA, Leonardo; ZABALA, Juan Pablo. La construcción de la utilidad social de la ciencia. Estrategias de los investigadores académicos en biotecnología frente al mercado. Bernal: Editorial de la Universidad Nacional de Quilmes, 2002.

VASEN, Federico. Los sentidos de la relevancia en la política científica. En: Revista Iberoamericana de Ciencia Tecnología y Sociedad, v. 7, n. 19, p. 11-46. Buenos Aires: Centro de Estudios sobre Ciencia, Desarrollo y Educación Superior (REDES), 2011.

VASEN, Federico. ¿Qué política científica para las humanidades? Espacios de crítica y producción, n. 48, p. 43-52. Buenos Aires: Editorial de la Facultad de Filosofía y Letras de la Universidad de Buenos Aires, 2012.

VESSURI, Hebe. Scientific Cooperation among Unequal Partners: the Strait-Jacket of the Human Resource Base. En: GAILLARD, J. (Ed.) En: Coopérations Scientifiques Internationales. Les Sciences Hors D’Occident au XXe. Siècle, p. 171-185. Paris: ORSTOM Editions, 1996.

VON SCHOMBERG, René "A vision of Responsible Research and Innovation". En OWEN, Richard; BESSANT,John; HEINTS, Maggie (Eds.) En: Responsible Innovation, p. 51-74. Londres: Wiley, 2013. 